\title{
Características Morfológicas e Produtivas de Clones de Batata Doce
}

\author{
Lázaro Kleber Assis Bevilaqua ${ }^{1}$, José Hortêncio Mota ${ }^{2 *}$, Geraldo Milanez de Resende ${ }^{3}$, Jony Eishi Yuri ${ }^{4}$
}

\section{Resumo}

Este estudo teve como objetivo avaliar características morfológicas e produtivas de cultivares de batata doce cultivadas em Jataí-GO. Foram avaliados 26 clones, empregando o delineamento em blocos casualizados com 3 repetições. Foram avaliadas 13 características morfológicas, sendo dez da parte aérea e três das raízes. Com base nos dados morfológicos obtidos foi estimada a correlação fenotípica entre todos os caracteres. Foi construído um dendrograma para verificar a influência da morfologia no agrupamento entre os clones. As características produtivas avaliadas foram: produtividade comercial e não comercial de raízes. Os dados de produção foram submetidos à análise de variância e as médias comparadas pelo teste de Scott-Knott a 5\% de probabilidade. Verificou-se que existe grande variabilidade fenotípica entre os clones avaliados, evidenciando uma significativa divergência com base nos descritores morfológicos. Em relação à produção, não houve diferença significativa para as produtividades comerciais dos clones avaliados.

Palavras-chave: Ipomoea batatas (L.) Lam. Descritores. Produtividade.

\section{Morphological and Productive Characteristics of Sweet Potato Clones}

\begin{abstract}
The aim of this study was to evaluate the morphological and productive characteristics of sweet potato cultivated in Jataí - Goiás, Brazil. Twenty six clones were evaluated using a randomized blocks design with three replications. Thirteen morphological characteristics were evaluated, ten of shoot and three of roots. The phenotypic correlations was estimated based on morphological characteristics. Clustering procedure was performed to verify the influence of morphology in the group between the clones. The productive characteristics evaluated were: productivity commercial and not commercial yield of roots. Production data were submitted to analysis of variance and means compared by the Scott-Knott test at 5\% probability. Great phenotypic variability among clones was observed, showing a significant difference based on morphological descriptors. There was no significant difference to the commercial yield of clones evaluated.
\end{abstract}

Keywords: Ipomoea batatas L. Descriptions. Yield.

\footnotetext{
${ }^{1}$ Universidade Federal de Goiás - Regional Jataí. Jataí, Goiás, Brasil. https://orcid.org/0000-0001-9166-9866

${ }^{2}$ Universidade Federal de Goiás - Regional Jataí. Jataí, Goiás, Brasil.

https://orcid.org/0000-0002-4403-0039

${ }^{3}$ Embrapa Semi-árido. Petrolina, Pernambuco, Brasil.

https://orcid.org/0000-0002-3295-8200

${ }^{4}$ Embrapa Semi-árido. Petrolina, Pernambuco, Brasil. https://orcid.org/0000-0003-1051-5940

*Autor para correspondência: hortenciomota@ufg.br
}

Recebido para publicação em 23 de outubro de 2019. Aceito para publicação em 06 de novembro de 2019

e-ISSN: 2447-6218 / ISSN: 2447-6218 / (C) 2009, Universidade Federal de Minas Gerais, Todos os direitos reservados. 


\section{Introdução}

A batata-doce (Ipomoea batatas (L.) Lam.) é uma cultura de importância econômicos e de social em muitos países. É cultivada em 243 países, com 76,1\% da produção localizado na Ásia, $19,5 \%$ na África 3,5\% nas Américas, 0,8\% na Oceania e $0,1 \%$ na Europa, sendo que a produção conjunta destes países situam-se em 1.848.670,95 toneladas cultivadas em uma área de $125.758,82$ hectares resultando em um produtividade média de 14,70 t ha-1 (FAO, 2017). Já a produção média do Brasil situa-se anualmente em torno de 776.285 t, obtidas em uma área plantada de 53.480 ha o que resultou em uma produtividade média de 14,51 t ha ${ }^{-1}$ (IBGE, 2017).

Entre as culturas de tubérculos e raízes, a batata-doce desempenha um papel importante na nutrição humana e animal, sendo de fácil cultivo e apresentando capacidade de adaptação a diferentes condições edafoclimáticas (Glato et al., 2017), com diferentes formas de aproveitamento, faz com que a cultura venha sendo difundido facilmente por diversas partes do mundo inclusive no Brasil. É considerada uma espécie rústica, que apresenta grande resistência a pragas, pouco exigente em fertilidade do solo, de fácil cultivo, ampla adaptação, alta tolerância à seca e baixo custo de produção (Montes et al., 2006; Monteiro et al., 2007).

No Brasil, encontra-se grande variabilidade genética para a espécie. Em praticamente todo o país são encontradas cultivares regionais, com características próprias (Cardoso et al., 2005). Uma vez que a base do melhoramento vegetal é a existência de variabilidade genética na espécie, onde os genótipos podem apresentar respostas diferentes à necessidade nutricional, resistência às pragas e doenças e outros, além de morfologia diferente, a caracterização de cada genótipo é de fundamental importância para que essa variabilidade possa ser utilizada de forma eficiente

Tabela 1 - Descrição nominal dos clones avaliados no desenvolvimento de novas cultivares, de acordo com as características das regiões para as quais serão destinadas.

Neste contexto, este estudo teve por objetivo caracterizar morfologicamente e avaliar o desempenho produtivo de 26 clones de batata doce cultivados nas edafoclimáticas do município de Jataí-GO.

\section{Avaliação da similaridade morfológica e produção dos clones de batata-doce}

O estudo foi conduzido na área experimental da Universidade Federal de Goiás (UFG)-Regional Jataí, situada nas coordenadas $17^{\circ} 66^{\prime} \mathrm{S}$ e $51^{\circ} 43^{\prime} \mathrm{O}$, a altitude média de $670 \mathrm{~m}$. O clima da região, segundo a classificação de Köppen, é do tipo Aw - tropical de savana e megatérmico com estações seca e chuvosa definidas. A temperatura média anual é de $23,7^{\circ} \mathrm{C}$ e a precipitação anual média é de 1644,9 mm (INMET, 2013).

Foram avaliados 26 clones de batata doce pertencentes ao banco de germoplasma de batata-doce da Regional Jataí da UFG (Tabela 1).

O experimento foi instalado em blocos casualizados, com 26 tratamentos e três repetições. As parcelas constaram de uma fileira de $2,1 \mathrm{~m}$ de comprimento com 7 plantas por parcela, utilizando o espaçamento entre plantas de 0,3 $\mathrm{m}$ e entre fileiras de $0,8 \mathrm{~m}$.

A área para o plantio foi preparada por meio de aração e gradagem, sendo posteriormente sulcado para formação das leiras, e aplicado $100 \mathrm{~g} \mathrm{~m}^{-2}$ de adubo 4-14-8. No plantio, foi utilizado ramas com oito entrenós, ficando enterrados três a quatro transversalmente sobre a leira, a uma profundidade de $10-15 \mathrm{~cm}$.

\begin{tabular}{|c|c|}
\hline Nome & Origem \\
\hline Arruba, BD 23, BD 54, Licori & Diamantina - MG \\
\hline Batata vermelha, Caruaru vermelha, Inhame branca, Sete semanas vermelha & Embrapa Semiárido - PE \\
\hline BJ 01, BJ 02 & Araguari - MG \\
\hline BJ 03 & Bebedouro - SP \\
\hline BJ 04 & Caratinga - MG \\
\hline BJ 05, BJ 06, BJ 07 & Jataí - GO \\
\hline BJ 08, BJ 09, BJ 10, BJ 11, BJ 12, BJ 13, BJ 14, BJ 15, BJ 16, BJ 17 & Taiúva - SP \\
\hline BJ 18 & Salvador - BA \\
\hline
\end{tabular}

A avaliação das características morfológicas foi realizada segundo os descritores recomendados por Huamán (1991). Foram avaliados 13 caracteres, sendo dez da parte aérea e três das raízes. As da parte aérea foram: forma da folha madura, tipo de lóbulo, número de lóbulos, forma do lóbulo central, tamanho da folha madura, cor da folha madura e imatura, comprimento do pecíolo e do entrenó e diâmetro do entrenó. As das raízes in natura foram: formato, cor externa da película, cor interna da polpa. 
As avaliações dos caracteres da parte aérea foram realizadas cinco meses após o plantio e os das raízes por ocasião da colheita (174 dias após o plantio). Conforme Cavalcante et al. (2010), os dados referentes às características de folhas e pecíolos foram obtidos da parte central das ramas, utilizando-se três folhas por planta e quatro plantas por parcela e, os das raízes, foram obtidos de todo material colhido de seis plantas úteis de cada parcela.

Com base nos dados morfológicos obtidos foi estimada a correlação fenotípica de Pearson entre todos os caracteres, seguindo-se da realização do teste $\mathrm{T}$ para verificar as correlações foram significativas.

Também foi construído um dendrograma para verificar a influência da morfologia no agrupamento entre os clones. Para o agrupamento dos caracteres morfológicos foi utilizada a distância euclidiana e o dendrograma de similaridade foi construído segundo o método da média aritmética ponderada (WPGMA).

As características produtivas avaliadas foram: produtividade comercial (obtida pela pesagem de todas as raízes com peso acima de $80 \mathrm{~g}$ e abaixo de $400 \mathrm{~g}$, segundo classificação apresentada por Silva et al. (2004) e produtividade não comercial (obtida pela pesagem das raízes que estão com algum dano ou que estejam abaixo ou acima do peso necessário para ser inserida com comercial).

Os dados de produtividade foram submetidos à análise de variância e as médias dos tratamentos foram comparadas utilizando-se o teste de Tukey a 5\% de probabilidade.

\section{Similaridade morfológica e produtividade}

Observou-se variações morfológicas entre os clones estudados. A variabilidade pode ser observada no formato das folhas que apresentou forma cordada (7,7\%), lanceolada $(11,5 \%)$, lobulada $(34,6 \%)$ e triangular $(46,2 \%)$. O tipo de lóbulo foi classificado como ausente $(15,4 \%)$, muito superficial $(30,8 \%)$, superficial $(23,1 \%)$, moderado $(19,2 \%)$ e profundo $(11,5 \%)$. Para a variável número de lóbulos, os clones foram divididos em quatro grupos distintos: folhas com um lóbulo $(15,4 \%)$, folhas com três $(57,7 \%)$, cinco $(23,1 \%)$ e sete $(3,8 \%)$ lóbulos. A forma do lóbulo central foi elíptico e lanceolado (7,7\% cada), dentado (15,4\%), semielíptico $(19,2 \%)$ e triangular (50\%). 7,7\% das folhas maduras apresentaram tamanho pequeno e grande, respectivamente, e $84,6 \%$ tamanho médio.

A cor da folha madura foi verde para a maioria $(80,8 \%)$, seguida pelas cores amarelo-verde e verde com bordas roxas $(7,7 \%$ cada) e verde com nervuras roxas na face $(3,8 \%)$. Quanto a cor da folha imatura, $30,8 \%$ apresentam cor verde, $26,9 \%$ cor amarelo-verde e verde com bordas roxas, respectivamente, $11,5 \%$ verde com nervuras roxas na face e $3,8 \%$ roxas em ambas as superfícies. O comprimento do pecíolo foi classificado com muito curto $(3,8 \%)$, curto $(53,8 \%)$ e intermediário $(42,3 \%)$. De modo geral, as folhas apresentaram comprimento de entrenó muito curto $(65,4 \%)$, seguido de comprimento curto $(30,8 \%)$ e longo $(3,8 \%)$. Já os diâmetros de entrenós foram finos $(46,2 \%)$ e intermediário $(46,2 \%)$.

Quanto ao formato das raízes, 44,9\% dos clones apresentaram raízes com formato desuniforme, com veias e bastante irregular; $25,6 \%$ com formato considerado bom, próximo de fusiforme, com algumas veias; $20,5 \%$ com raízes grandes, com veias e rachaduras, indesejáveis comercialmente; e 9,0\% apresentaram raízes totalmente fora dos padrões comerciais, muito irregulares e deformadas, com muitas veias e rachaduras.

A cor externa da película predominante nos clones avaliados foi rosada $(38,5 \%)$, seguida de roxa $(30,8 \%)$, branca $(23,1 \%)$ e creme $(7,7 \%)$. Quanto a cor interna da polpa, ocorreu predomínio da cor creme $(65,4)$ e ocorrência das cores branca $(23,1 \%)$, amarela $(7,7 \%)$ e roxa $(3,8 \%)$.

Essas observações são corroboradas por Moulin et al. (2014), que estudando características morfológicas de 46 acessos de batata doce coletados em propriedades rurais e estabelecimentos comerciais da região Norte do Estado do Rio de Janeiro, verificaram alta variabilidade fenotípica dos genótipos de batata-doce estudados.

De acordo com a matriz de correlação fenotípica para as variáveis avaliadas (Tabela 2), o tipo de lóbulo da folha (TL) apresentou forte correlação com a forma do lóbulo central (FLC), sendo igual a 0,79, e também com a forma de folha madura (FFM), igual a 0,67 , e com o número de lóbulo (NL), igual a 0,59 , sendo altamente significativo.

Com base nas médias dos dados morfológicos obtidos a partir dos clones, obteve-se a matriz de distâncias euclidianas, a partir da qual se gerou o dendrograma (Figura 1). Observa-se que houve a formação de dois grupos distintos com subdivisões. $\mathrm{O}$ primeiro grupo reúne os seguintes clones: arruba, BJ 02, BJ 04, BJ 06, BJ 18, Inhame branca, Licori e Sete Semanas Vermelha. Os clones reunidos no segundo grupo foram: Batata Vermelha, BD 23, BD 54, BJ 01, BJ 03, BJ 05, BJ 07, BJ 08, BJ 09, BJ 10, BJ 11, BJ 12, BJ 13, BJ 14, BJ 15, BJ 16, BJ 17 e Caruaru Vermelha.

Os resultados obtidos confirmam a alta variabilidade morfológica entre os clones de batata doce (Figura 1), com ausência de fenótipos iguais, reforçando, conforme Neiva et al. (2011), a diversidade advinda dos parentais. Observa-se pelo dendrograma de dissimilaridade morfológica, que os clones mais similares morfologicamente são BJ 01 e BJ 15 e BJ 03 e BJ 12 e os mais dissimilares são Arruba e BJ 11. 
Bevilaqua, L. K. A. et al.

Tabela 2 - Matriz de correlação fenotípica para as variáveis avaliadas

\begin{tabular}{|c|c|c|c|c|c|c|c|c|c|c|c|c|c|}
\hline & FFM & TL & NL & FLC & TFM & CFM & CFI & CP & CE & $\mathrm{DE}$ & FR & CIP & CEP \\
\hline FFM & 1 & $0,67^{*}$ & 0,21 & $0,51^{*}$ & $-0,10$ & $-0,17$ & $-0,19$ & $-0,02$ & $-0,16$ & $-0,32$ & $-0,21$ & 0,13 & 0,02 \\
\hline TL & & 1 & $0,59^{*}$ & $0,79 *$ & $-0,09$ & $-0,13$ & $-0,21$ & 0,34 & $-0,17$ & $-0,06$ & $-0,10$ & $-0,19$ & $-0,18$ \\
\hline NL & & & 1 & 0,35 & 0,14 & $-0,11$ & $-0,14$ & 0,24 & $-0,05$ & 0,20 & $-0,11$ & 0,02 & 0,09 \\
\hline FLC & & & & 1 & $-0,33$ & $-0,12$ & $-0,19$ & 0,33 & $-0,26$ & $-0,14$ & $-0,11$ & $-0,17$ & $-0,19$ \\
\hline TFM & & & & & 1 & 0,14 & 0,06 & 0,18 & 0,35 & $0,39 *$ & $-0,16$ & 0,11 & 0,09 \\
\hline CFM & & & & & & 1 & $0,48^{*}$ & $-0,11$ & 0,08 & 0,26 & $-0,11$ & $-0,15$ & $-0,12$ \\
\hline CFI & & & & & & & 1 & $-0,40^{*}$ & $-0,01$ & 0,24 & $-0,08$ & $-0,16$ & $-0,04$ \\
\hline CP & & & & & & & & 1 & 0,14 & 0,36 & $-0,09$ & 0,03 & $-0,32$ \\
\hline $\mathrm{CE}$ & & & & & & & & & 1 & 0,36 & $-0,09$ & $-0,19$ & 0,06 \\
\hline DE & & & & & & & & & & 1 & $-0,01$ & $-0,10$ & $-0,31$ \\
\hline FR & & & & & & & & & & & 1 & $-0,30$ & 0,08 \\
\hline CIP & & & & & & & & & & & & 1 & 0,23 \\
\hline CEP & & & & & & & & & & & & & 1 \\
\hline
\end{tabular}

Em que: $\mathrm{FFM}$ = forma de folha madura; TL = tipo de lóbulo; $\mathrm{NL}=$ número de lóbulos; FLC $=$ forma do lóbulo central; TFM =tamanho de folha madura; $\mathrm{CFM}=$ cor de folha madura; $\mathrm{CFI}=$ cor de folha imatura; $\mathrm{CP}=$ comprimento do pecíolo; $\mathrm{CE}=$ comprimento do entrenó; $\mathrm{DE}=$ diâmetro do entrenó; FR = formato da raiz; CIP = cor interna da polpa e CEP = cor externa da película.

*Significativo a $5 \%$ pelo teste $\mathrm{T}$.

Moulin et al. (2012), avaliando 46 acessos de batata-doce em propriedades rurais situadas na região Norte do Estado do Rio de Janeiro, também verificaram um elevado número de classes fenotípicas ao avaliar as características morfológicas dos mesmos.

Martins et al. (2012), avaliando a variabilidade fenotípica e a divergência genética entre 50 clones de batata-doce no estado do Tocantins, identificaram oito grupos geneticamente disjuntos concluindo que há uma alta variabilidade morfológica nos clones avaliados, o que foi verificado no experimento.

Resultados similares foram obtidos por Neiva et al. (2011) que, avaliando 65 clones da coleção do banco de germoplasma da UFVJM, também observaram pelo dendrograma de dissimilaridade morfológica que os clones apresentaram ampla variabilidade morfológica.

As características morfológicas podem variar devido à plasticidade das características (Price et al., 2003) sendo que o ambiente pode afetar as características fenotípicas (Yada et al., 2010). Dessa forma, as comparações entre estudos de diversidade morfológica são difíceis (Glato et al, 2017).
Em relação à produtividade não comercial, houve foram observadas diferenças entre os clones (Tabela 3) apenas para a característica produtividade não comercial de raízes a qual apresentou uma variação de $72,5 \%$ do clone menos produtivo $\left(10,6 \mathrm{t} \mathrm{ha}^{-1}\right)$ para o mais produtivo $\left(38,9\right.$ tha $\left.^{-1}\right)$.

Já para produtividade comercial média de raízes não houve diferença significativa entre os clones.

Verificou-se grande variação nas produtividades, as quais estão estreitamente relacionadas ao material genético, ao local de cultivo, à época de plantio. Andrade Júnior et al. (2012), avaliando 12 clones de batata doce em Diamantina-MG, obteve médias de produção comercial de 8 a 29,5 t ha $^{-1}$, variação de $72,9 \%$.

\section{Conclusão}

Existe grande variabilidade fenotípica entre os 26 clones avaliados, evidenciando uma significativa divergência com base nos descritores morfológicos.

Em relação à produção, não houve diferença significativa das produtividades comerciais dos clones avaliados. 
Figura 1 - Dendrograma de dissimilaridade morfológica de 26 clones de batata-doce, estabelecido pelo método WPGA utilizando-se a distância euclidiana, com base em 13 caracteres morfológicos

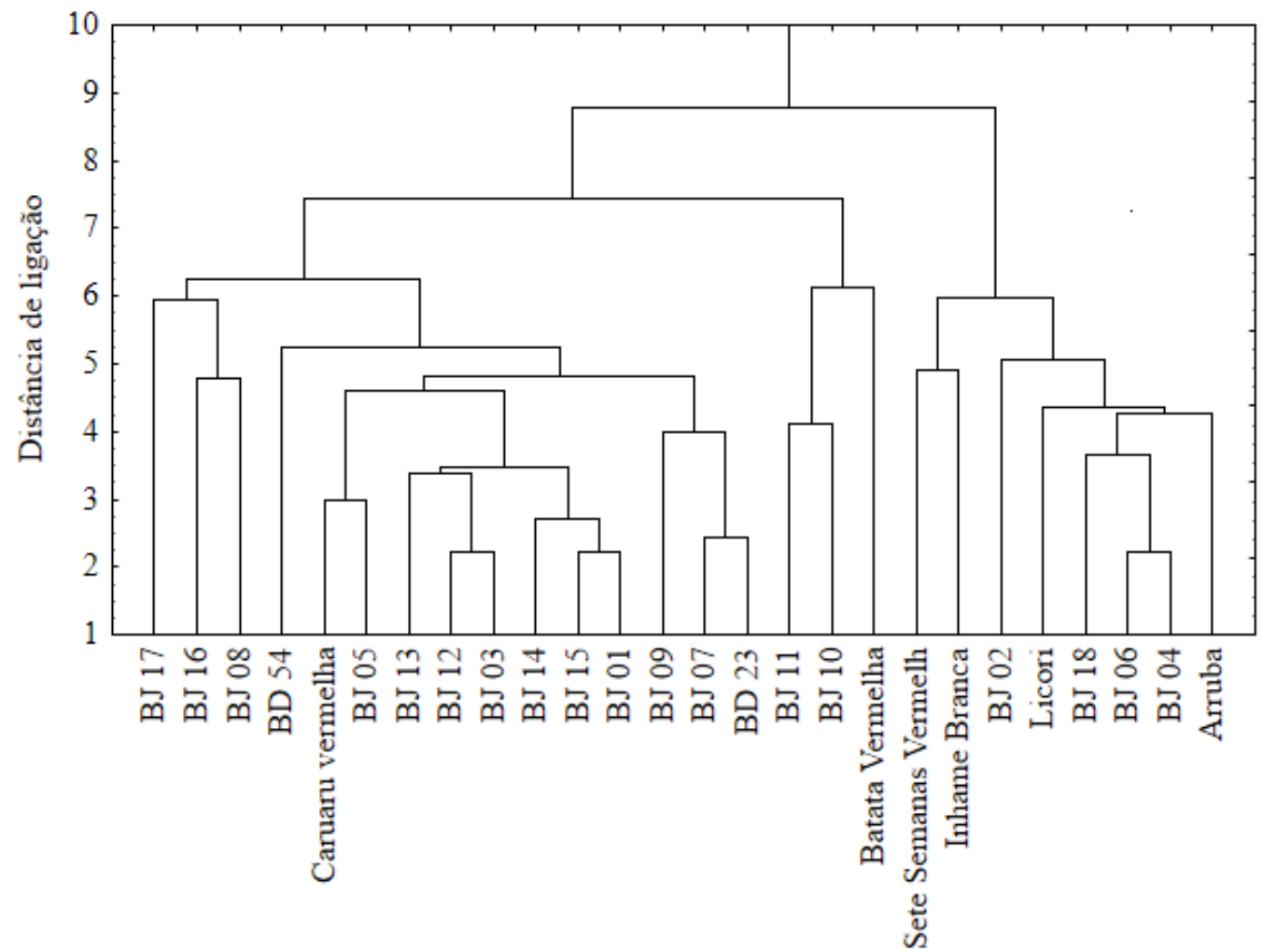

Tabela 3 - Valores médios de produtividade não comercial e comercial de raízes

\begin{tabular}{|c|c|c|}
\hline Clone & $\begin{array}{l}\text { Produtividade não comercial } \\
\qquad\left(\mathrm{t} \mathrm{ha}^{-1}\right)\end{array}$ & $\begin{array}{l}\text { Produtividade comercial } \\
\qquad\left(\mathrm{t} \mathrm{ha} \mathrm{a}^{-1}\right)\end{array}$ \\
\hline BJ 15 & 38,9 a & $5,4 \mathrm{a}$ \\
\hline Batata Vermelha & $35,9 \mathrm{ab}$ & $6,1 \mathrm{a}$ \\
\hline BJ 07 & $35,5 \mathrm{a} \mathrm{b}$ & 5,7 a \\
\hline Inhame Branca & $31,1 \mathrm{a} \mathrm{b} \mathrm{c}$ & 6,4 a \\
\hline BJ 03 & $30,2 \mathrm{a} \mathrm{b} \mathrm{c}$ & $2,3 \mathrm{a}$ \\
\hline Caruaru Vermelha & 29,0 a b c & 5,7 a \\
\hline BJ 05 & $28,62 \mathrm{a} \mathrm{b} \mathrm{c}$ & $5,6 \mathrm{a}$ \\
\hline BJ 13 & $27,5 \mathrm{abc}$ & $3,8 \mathrm{a}$ \\
\hline Sete Semanas Vermelha & 27,3 a b c & $2,4 \mathrm{a}$ \\
\hline BJ 01 & $25,2 \mathrm{a} \mathrm{b} \mathrm{c}$ & 4,5 a \\
\hline BJ 14 & $24,7 \mathrm{a} \mathrm{b} \mathrm{c}$ & $6,4 \mathrm{a}$ \\
\hline BJ 11 & $23,4 \mathrm{abc}$ & $5,0 \mathrm{a}$ \\
\hline BJ 08 & $23,3 \mathrm{a} \mathrm{b} \mathrm{c}$ & $1,1 \mathrm{a}$ \\
\hline BJ 18 & 22,9 a b c & 4,6 a \\
\hline BJ 09 & $22,7 \mathrm{a} b \mathrm{c}$ & $4,1 \mathrm{a}$ \\
\hline BJ 17 & $22,4 \mathrm{a} \mathrm{b} \mathrm{c}$ & $4,6 \mathrm{a}$ \\
\hline
\end{tabular}


Bevilaqua, L. K. A. et al.

\begin{tabular}{|c|c|c|}
\hline Clone & $\begin{array}{l}\text { Produtividade não comercial } \\
\qquad\left(\mathrm{t} \mathrm{ha}^{-1}\right)\end{array}$ & $\begin{array}{l}\text { Produtividade comercial } \\
\qquad\left(\mathrm{t} \mathrm{ha} \mathrm{h}^{-1}\right)\end{array}$ \\
\hline BJ 02 & 19,7 a b c & $2,9 \mathrm{a}$ \\
\hline BJ 04 & 17,9 a b c & $3,1 \mathrm{a}$ \\
\hline Licori & 17,7 a b c & $4,2 \mathrm{a}$ \\
\hline BJ 12 & 17,6 a b c & $3,7 \mathrm{a}$ \\
\hline BJ 16 & $16,5 \mathrm{abc}$ & $1,6 \mathrm{a}$ \\
\hline BJ 10 & $14,3 \mathrm{~b} \mathrm{c}$ & $1,3 \mathrm{a}$ \\
\hline BJ 06 & $13,2 \mathrm{~b} \mathrm{c}$ & $5,8 \mathrm{a}$ \\
\hline BD 54 & $12,8 \mathrm{~b} \mathrm{c}$ & $2,1 \mathrm{a}$ \\
\hline BD 23 & $12,0 \mathrm{~b} \mathrm{c}$ & $1,8 \mathrm{a}$ \\
\hline Arruba & $10,6 \mathrm{c}$ & $2,7 \mathrm{a}$ \\
\hline CV (\%) & 32,7 & 28,9 \\
\hline Média & 23,1 & 4,0 \\
\hline
\end{tabular}

Médias seguidas da mesma letra na coluna não diferem entre ao nível de $5 \%$ pelo teste de Tukey.

\section{Referências}

Andrade Júnior, V. C.; Viana, D. J. S.; Pinto, N. A. V. D.; Ribeiro, K. G.; Pereira, R. C.; Neiva, I. P.; Azevedo, A. M.; Andrade, P. C. R. 2012. Características produtivas e qualitativas de ramas e raízes de batata-doce. Horticultura Brasileira, 30: 584-589. Doi: http://dx.doi.org/10.1590/ S0102-05362012000400004.

Cardoso, A. D.; Viana, A. E. S.; Ramos, P. A. S.; Matsumoto, S. N.; Amaral, C. L. F.; Sediyama, T.; Morais, O. M. 2005. Avaliação de clones de batata-doce em Vitória da Conquista. Horticultura Brasileira, 23: 911-914. Doi: http://dx.doi.org/10.1590/S0102-05362005000400009.

Cavalcante, M.; Ferreira, P. V.; Paixão, S. L.; Costa, J. G.; Pereira, R. G.; Madalena, J. A. 2010. Desempenho agronômico, dissimilaridade genética e seleção de genitores de batata doce para hibridização. Revista Brasileira de Ciências Agrárias, 5: 485-490. Doi: http://dx.doi. org/10.5039/agraria.v5i4a816.

FAO - Food and Agriculture Organization of the United Nations. 2016. Faostat. Disponível em: http://www.fao.org/faostat/en/\#data/QC.

Glato, K.; Aidam, A., Kane, N. A.; Bassirou, D.; Couderc, M.; Zekraoui, L.; Scarcelli, N.; Barnaud, A.; Vigouroux, Y. Structure of sweet potato (Ipomoea batatas) diversity in West Africa covaries with a climatic gradiente. PLoS ONE, 12: e0177697. Doi: https://doi.org/10.1371/ journal.pone.0177697.

Huamán, Z. 1991. Descriptors for sweet potato. Roma: IBPGR.

INMET - Instituto Nacional de Meteorologia. 2013. BDMEP - Banco de Dados Meteorológicos para Ensino e Pesquisa: Série Histórica - Dados Diários de 01/01/1982 a 31/12/2012 Estação: 83464 - Jataí - GO. Disponível em: http://www.inmet.gov.br/portal/index.php?r=bdmep/ bdmep.

IBGE - Instituto Brasileiro de Geografia e Estatística. Produção agrícola Municipal: Disponível em https://www.ibge.gov.br/estatisticasnovoportal/economicas/agricultura-e-pecuaria/9117-producao-agricolamunicipal-culturas-temporarias-e-permanentes.html $?=\& \mathrm{t}=$ resultados.
Martins, E. C. A.; Peluzio, J. M.; Coimbra, R. R.; Oliveira Junior, W. P. 2012. Variabilidade fenotípica e divergência genética em clones de batata-doce no estado do Tocantins. Revista Ciência Agronômica, 43: 691-697.

Monteiro, A. B.; Massaroto, J. A.; Gasparino, C. F.; Silva, R. R.; Gomes, L. A. A.; Maluf, W. R.; Silva Filho, J. C. 2007. Silagens de cultivares e clones de batata-doce para alimentação animal visando sustentabilidade da produção agrícola familiar. Revista Brasileira de Agroecologia, 2: 978-981.

Montes, S. M. N. M.; Firetti, R.; Golla, A. R.; Tarsitano, M. A. A. 2006. Custos e rentabilidade da batata-doce (Ipomoea batatas L.) na Região Oeste do Estado de São Paulo. Informações Econômicas, São Paulo, v. 36 , p. $15-23$.

Moulin, M. M.; Rodrigues, R.; Gonçalves, L. S. A.; Sudré, C. P.; Santos, M. H.; Silva, J. R. P. 2012. Collection and morphological characterization of sweet potato landraces in north of Rio de Janeiro state. Horticultura Brasileira, 30: 286-292. Doi: http://dx.doi.org/10.1590/S010205362012000200017.

Moulin, M. M.; Bento, C. S.; Santos Júnior, A. C.; Rodrigues, R. 2014. Caracterização de acessos de batata-doce baseado em características morfológicas. Perspectivas online: Biologia \& Saúde, 13: 23-26. Doi: https://doi.org/10.25242/8868413201451.

Price, T. D.; Qvarnstrom, A.; Irwin, D. E. 2003. The role of phenotypic plasticity in driving genetic evolution. Proceeding of the Royal Society of the London, 270: 1433-1440. Doi: https://doi.org/10.1098/ rspb.2003.2372.

Neiva, I. P.; Andrade Júnior, V. C.; Viana, D. J. S.; Figueiredo, J. A.; Mendonça Filho, C. V.; Parrella, R. A. C; Santos, J. B. 2011. Caracterização morfológica de acessos de batata-doce do banco de germoplasma da UFVJM, Diamantina. Horticultura Brasileira, 29: 537-541. Doi: http:// dx.doi.org/10.1590/S0102-05362011000400016. 
Características Morfológicas e Produtivas de Clones de Batata Doce

Silva, J. B. C.; Lopes, C. A.; Magalhães, J. S. 2004. Cultura da batatadoce (Ipomoea batatas L.). Brasília: EMBRAPA-CNPH. Disponível em: http://www.cnph.embrapa.br/sistprod/batatadoce.

Yada, B.; Tukamuhabwa, P.; Wanjala, B.; Kim, D. J.; Skilton, R.; Alajo, A.; Mwanga, R. 2010. Characterization of Ugandan Sweet potato
Germplasm Using Fluorescent Labeled Simple Sequence Repeat Markers. HortScience, 45: 225-230. Doi: http://dx.doi.org/10.21273/ hortsci.45.2.225. 